(Original)

\title{
Anatomical structure of the subcutaneous tissue on the anterior surface of human thigh
}

\author{
By \\ Teruki ISHIDA ${ }^{1}$, Kyoko TAKEUCHI ${ }^{2}$, Shogo HAYASHI ${ }^{1}$, Shinichi KAWATA ${ }^{1}$, \\ Naoyuki HATAYAMA ${ }^{1}$, Ning QU $^{1}$, Masakazu SHIBATA ${ }^{3}$, and Masahiro ITOH $^{1}$ \\ ${ }^{1}$ Department of Anatomy, Tokyo Medical University, 6-1-1 Shinjuku, Shinjuku-ku, Tokyo, Japan \\ ${ }^{2}$ Department of Health Science, Graduate School of Teikyo Heisei University, \\ 2-51-4 Higashi-ikebukuro, Toshima-ku, Tokyo, Japan \\ ${ }^{3}$ Faculty of Health and Social Services, Kanagawa University of Human Services, \\ 1-10-1 Heisei-cho, Yokosuka-city, Kanagawa, Japan
}

-Received for Publication, December 25, 2014-

Key Words: Subcutaneous tissue, Fascia, Anterior thigh, Gross dissection, Ultrasonic imaging

\begin{abstract}
Summary: The anterior thighs of 16 limbs from eight donated cadavers were observed using ultrasonic imaging and gross dissection with a specific focus on the subcutaneous tissue, which is considered an auxiliary element of locomotion. On ultrasonic imaging, the subcutaneous tissue was found to comprise multiple layers. The number of layers gradually decreased on progressing distally in the thigh. On gross dissection, a lot of fatty tissue and loose multiple laminar structures were observed in the medial and proximal areas. However, on progressing distally, these layers thinned out and became less fatty. Cutaneous nerves were apparent among the layers below the dermis. In addition, there were many associated fiber bundles between the dermis and muscle fascia, some of which appeared to be so-called skin ligaments that run through the subcutaneous tissue perpendicularly from the fascia to dermis, accompanying cutaneous nerve fibers and blood vessels. While identifying the peripheral cutaneous nerve fibers, several anastomosing rami and neural networks were recognized. These observations suggest that skin ligaments could be elements regulating motor restriction during muscular movement.
\end{abstract}

\section{Introduction}

The skin $^{12) 3)}$ is classified into the epidermis, dermis, and subcutaneous tissue(4)5)67), and is considered as a biological defense system and a sensory $\operatorname{organ}^{8) 9}$. The subcutaneous tissue, which envelops the overall musculature with the exception of cutaneous muscles, provides for the passage of cutaneous nerves, blood vessels, and lymph vessels and plays a role in connecting the dermis and fascia of the muscles ${ }^{10) 11}$.

In the field of rehabilitation and sports, the skin has recently gained significance as a member of the locomotorium that assists muscular movement ${ }^{12-22) .}$

Some researchers have reported that subcutaneous tissue moves relative to the fascia of the muscle 232425)26)27). Other reports advocate that a conditioning method involving skin movement with manual therapy facilitates pain reduction and functional improvement ${ }^{28)}$. According to Ishii et al. ${ }^{29)}$, the functional role of the fascial connections is to facilitate different types of contractions simultaneously in multiple muscles to act more efficiently to generate sufficient muscle output.

The function of the fascia and subcutaneous tissue as a part of the locomotor apparatus has received widespread attention; however, there are only a few articles describing the morphological relation between the skin and muscular movement ${ }^{30}$. To our knowledge, Nash et al. ${ }^{31)}$ have histologically presented the existence of the skin ligaments between the skin and muscle fascia that are extensively present in the face, hands, feet, and breast tissue. Nakajima et al. have classified the connective tissue into two layers - the protective adipofascial system (PAFS) and lubricant adipofascial system (LAFS) - and reported that these layers have suitably modulated thicknesses across different parts of the body although they did not describe the specific details for the thigh muscles ${ }^{32}$.

This cadaveric study aims to identify the connecting structures between the dermis and subcutaneous tissue 
as well as the fascial connections between the subcutaneous tissue and muscle fascia, with a focus on the anterior surface of human thigh, extending from the inguinal region to the knee joint.

\section{Materials and Methods}

The anterior aspect of the thighs in 16 limbs from eight donated cadavers ( 3 men and five women; mean age, $84.9 \pm 10.2$ years) was observed. The cadavers were fixed with $10 \%$ formalin solution $(3.5 \%-3.8 \%$ formaldehyde). The observational area of each thigh was defined by margins (Fig. 1) as follows: 1) the lateral margin, from the anterior superior iliac spine to the lateral side of the knee via the greater trochanter; 2) the superior margin, comprising the inguinal ligament; 3 ) the medial margin, from the pubic epiphysis of the inguinal ligament to the medial side of the knee; and 4) the inferior margin, marked by the superior margin of the patella.

\section{Ultrasonic imaging}

The anterior aspect of both thighs from one female cadaver (78 years) was observed with an ultrasonic imaging device (UGEO PT60A, Samsung, Korea) to evaluate the structures ranging from the skin to the fascia before dissection. As tiny wrinkles on the skin surface of the fixed cadaver were harder than those observed in living subjects, a thicker gel coat was applied to the skin surface to facilitate imaging.

The observational area was further divided into four (approximately $10 \mathrm{~cm} \times 5 \mathrm{~cm}$ ) regions: the superior medial, the superior lateral, the inferior medial, and the inferior lateral regions (Fig. 1). The points from which characteristic images were obtained were marked on the skin surface and then anatomically observed.

\section{Anatomical observation}

The skin over the anterior surface of the thigh was routinely incised with a scalpel and forceps to expose the subcutaneous tissue, including fat, which was then further dissected for observation.

Tissues in the layers from the subcutaneous tissue to the fascia lata were bluntly dissected with forceps and fingers to prevent the disruption of the natural stratification of the subcutaneous tissue and fibrous structure. Thereafter, fatty tissues deposited along the course of the blood vessels and nerves running through the surface layer were carefully removed, and the deeper layer was surgically incised.

In the left anterior thigh of a male cadaver (74 years), the subcutaneous tissue including the connection across layers was excised and treated with the silver impregnation method established by Kimura and Takahashi ${ }^{33)}$ to determine the presence/absence of nerve fibers.

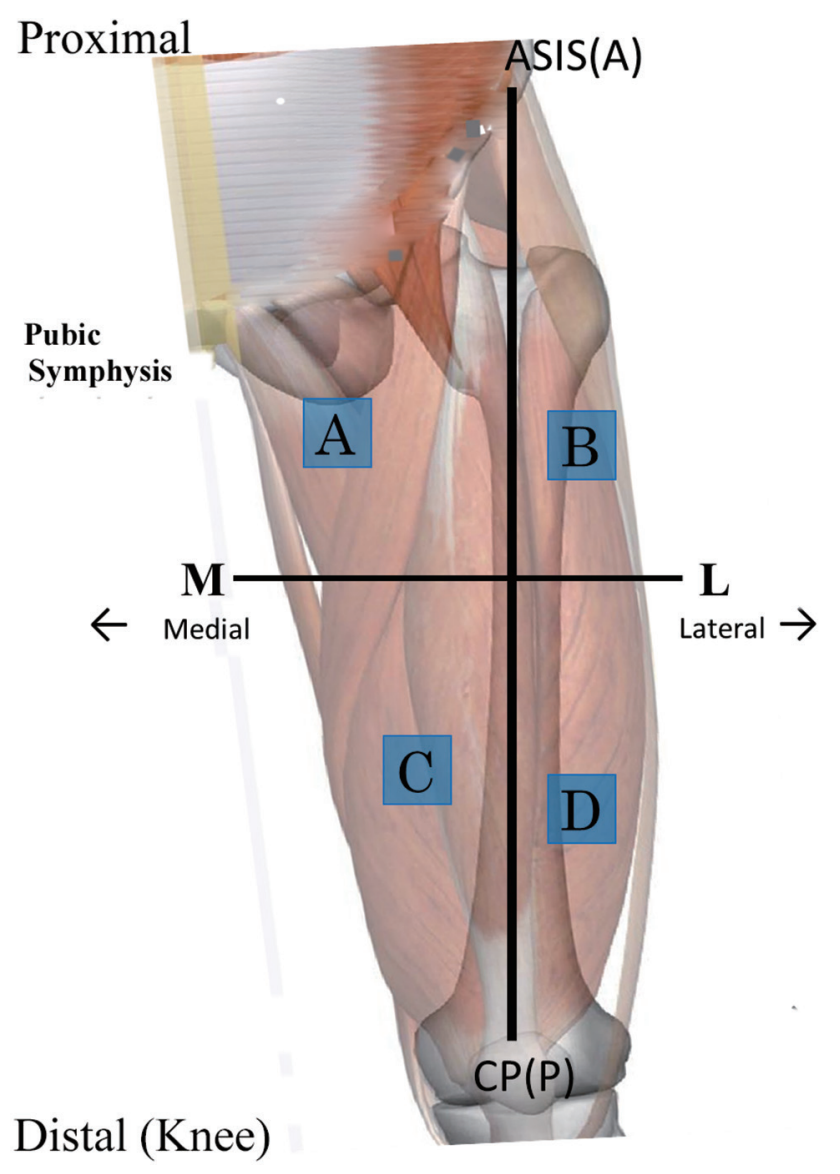

Fig. 1. Anterior surface of the left thigh. The observational area is demarcated by a dotted line. A black straight line (A-P line) connects the anterosuperior iliac spine (ASIS) with the center of the patella $(\mathrm{CP})$, dividing the thigh into medial and lateral regions $\mathrm{A}$ black transverse (horizontal) line (M-L line) passes perpendicularly through the midpoint of the A-P line, forming a transversal axis. The M-L line divides the thigh into upper (proximal) and lower (distal) sites. The blue squares A, B, C, and D in the figure correlate with the regions $\mathrm{A}, \mathrm{B}, \mathrm{C}$, and $\mathrm{D}$ observed on ultrasonic images in Fig. 4. The figure was transferred and modified from Kin-Nabi Puremium (Roundflat Co. Ltd., Japan) under authorization.

\section{Results}

Ultrasonic imaging revealed several discontinuous layers in the superomedial region (femoral triangle) of the thighs. The subcutaneous tissue presented a discontinuous multilayered structure (Fig. 2A). Several layers near the superolateral region of the thigh comprised the insertion of the iliotibial tract and surface layer near the tensor fascia lata serially (Fig. 2B). The subcutaneous tissue had a multilayered structure, and the low-echo layers were thicker than the high-echo layers.

The number of layers decreased to one or two in the anteroinferior regions of the thigh (Figs. 2C and D). In the inferomedial region, only a slight stratification 

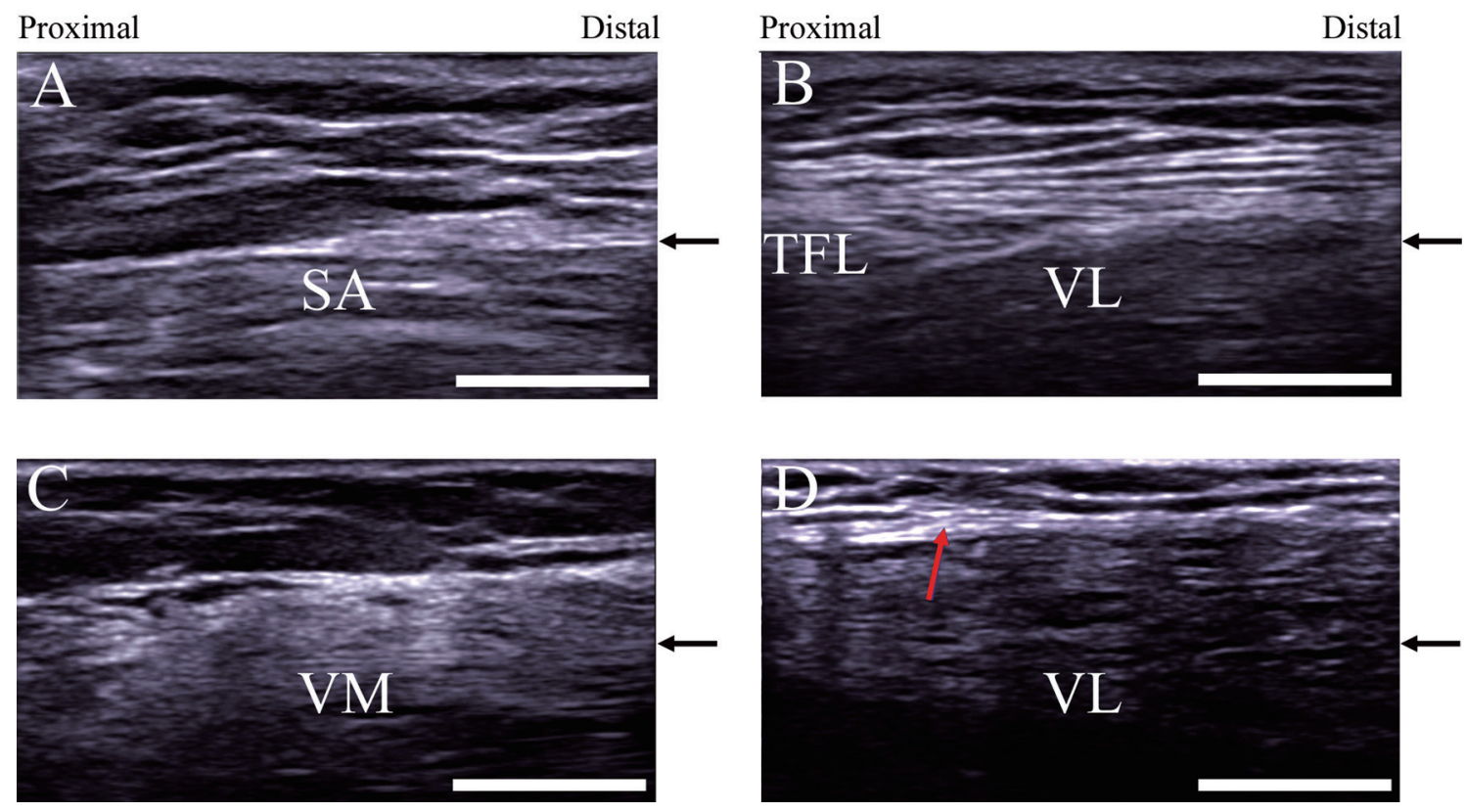

Fig. 2. Ultrasonic imaging of the subepidermal regions of the cadaveric thigh. Panels A, B, C, and D correspond to areas A, B, C, and D in Fig. 1. Ultrasonic image of A: the superior medial region, the subcutaneous tissue layers show a discontinuous multilayered structure; B: the superolateral region, the subcutaneous tissue with relatively slight fatty tissue distribution shows a multilayered structure; C: the inferomedial region, where only slight stratification is shown in the fasciae but thick fat layers are observed; and D: the inferolateral region, where fatty tissue is relatively less, and the number of the layers of the subcutaneous tissue are also smaller. The red arrow shows the tightly bound connection corresponding to Fig.4. Sa: sartorius, TFL: tensor fascia lata, VL: vastus lateralis, Vm: vastus medialis; focal depth $(\rightarrow): 10 \mathrm{~mm}$. Scale bar: $10 \mathrm{~mm}$

was evident in the subcutaneous tissue although the fat layer was relatively thick. Areas where the connections between the fascia lata and dermis were deeper than at other sites (Fig. 2D).

The number of layers in the subcutaneous tissue of the thigh progressively decreased on moving further distally, and cutaneous nerve distribution was macroscopically confirmed in each layer. On further dissection for the removal of fatty tissue, several layers were confirmed in the vicinity of the inguinal region (Fig. 3). A complex fascial structure comprising several layers was observed in the vicinity of the sartorius, with cutaneous nerve fibers and cutaneous veins interspersed between the layers of the subcutaneous tissue.

There were connective fibers with various stratification patterns between the subcutaneous tissue and fascia lata, and these were perpendicular to the thigh (Fig. 4). At the level of the rectus femoris, tightly bound connections between the subcutaneous tissue layer and surface layer of the fascia lata were observed (Fig. 4). At some such connections, the connective tissue fibers with nerves emerged multidirectionally toward the dermis (Fig. 4).

The cutaneous nerve fibers in these tightly bound subcutaneous regions were observed as black lines in the connective tissue of the subcutaneous tissue with the silver impregnation method. The nerve fibers were

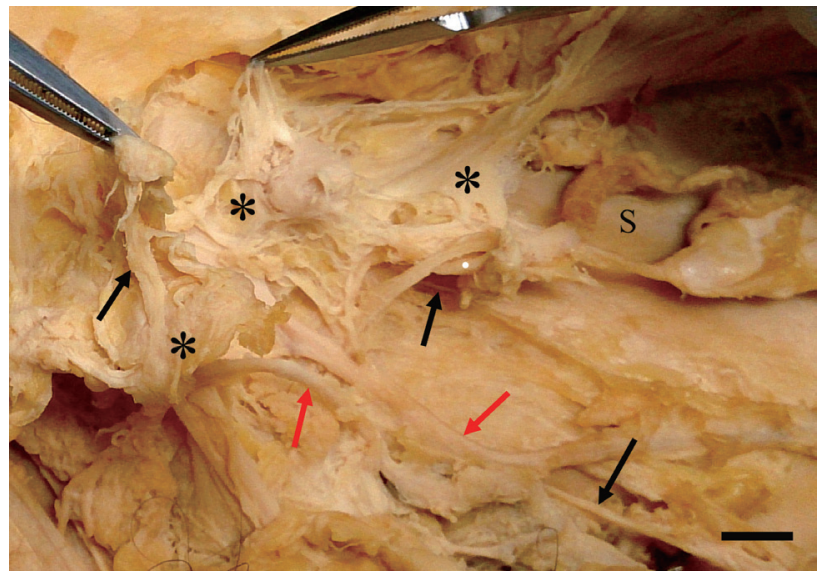

Fig. 3. Subcutaneous tissues surrounding the left inguinal region. The subcutaneous tissue comprising several layers is observed in the complex structure of fasciae (asterisks) in the vicinity of the sartorius muscle (S). Cutaneous nerve fibers (black arrows) and cutaneous veins (red arrows) run between the layers of the subcutaneous tissue. Scale bar: $1 \mathrm{~cm}$

distributed in the connective tissue, and peripheral parts of these cutaneous nerves created anastomosing rami (Fig. 5). 


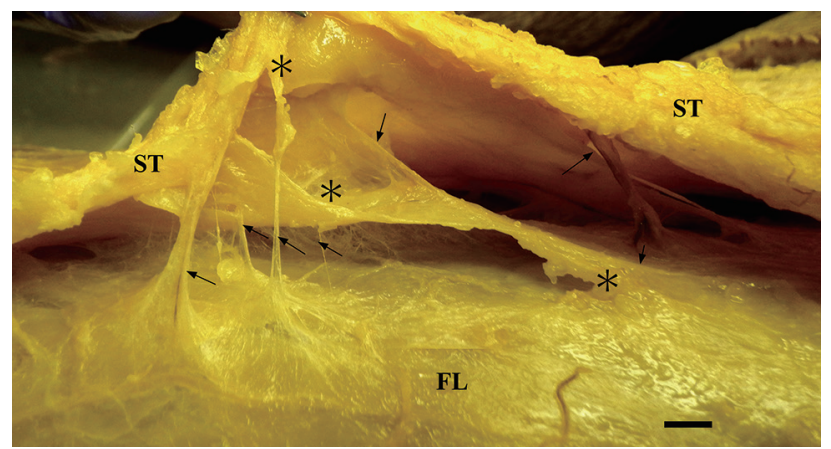

Fig. 4. Tightly bound connections. The tightly bound connection (asterisk) between the subcutaneous tissue (ST) and fascia lata (FL) at the level of the rectus femoris. Arrows indicate sites where connective tissue fibers with nerves emerge in multiple directions toward the dermis. These structures were observed on echography (Fig. 2D; arrow). Scale bar: $1 \mathrm{~cm}$

\section{Discussion}

There are different descriptions of the skin and fascia. In this article, the skin was defined as including the epidermis, dermis, and subcutaneous tissue ${ }^{45) 67)}$, and fascia was defined as muscle fascia or fascia lata without further categorization into superficial or deep fascia. Our present observations focused on the stratification of subcutaneous tissues in the anterior surface of the thigh as well as the connections of the subcutaneous tissues and fasciae in this region.

Because loose connective tissue fibers in the subcutaneous tissue play a role in the connection between the dermis and muscle fascia, it has been considered to transmit muscular movements to the skin. Although the skin and fasciae have been extensively evaluated from the functional aspect, the structural association of the subcutaneous tissue with the fascia has been discussed only from an anatomical standpoint. This may be because, anatomically, the connective tissue has been considered to be no more than a supportive structure that serves as capsules for organs and passages for vascular nerves. However, in recent years, the skin has been considered to have a functional role as an auxiliary factor in the locomotorium, and also as a restrictive element. Some reports have shown that manual stimulation through the skin is effective in functional improvement and pain reduction during rehabilitation and conditioning ${ }^{34) 35) 36)}$; however, the details of the pertinent morphology have not been discussed. In this study, we attempted to clarify the connecting structure between the dermis and subcutaneous tissue as well as fascial connections of the subcutaneous tissue and muscle fascia.

In the subcutaneous tissue of the thigh, stratification

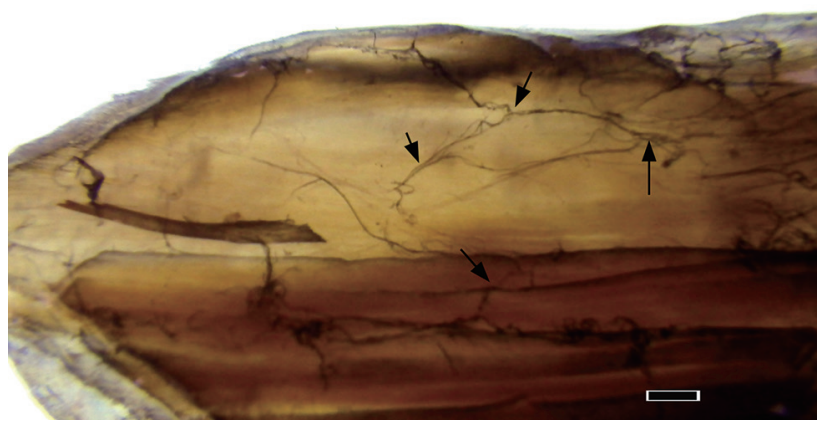

Fig. 5. Tightly bound subcutaneous region stained using the silver impregnation method. Nerve fibers (arrows) are distributed in the subcutaneous tissue. The peripheral parts of cutaneous nerves create anastomosing rami. Scale bar: $1 \mathrm{~mm}$

revealed that the structure comprised several layers. The number of layers decreased gradually on moving distally in the thigh, Moreover, ultrasonic imaging also revealed that the number of layers progressively decreased (Fig. 2 ). The anterior surface of the thigh is an area comprising the main distribution of the cutaneous rami of the femoral nerves. After the cutaneous rami branch off from the femoral nerves in the deeper regions of the femoral triangle, they further branch out into thinner nerves that pass through the subcutaneous tissue to emerge beneath the dermis. Our results suggest that the cutaneous rami emerging beneath the dermis in the area distal to the body trunk descend into the deeper layers of the subcutaneous tissue, whereas these nerves pass through the subcutaneous tissue in the area proximal to the body trunk.

Cutaneous nerves were distributed in the surface layer of the subcutaneous tissue and among other layers. Fibrous connections between the epidermis/dermis and subcutaneous tissues were confirmed on macroscopic anatomical dissection and visualization. Because cutaneous nerves provide connections toward the skin, it may be inferred that the subcutaneous tissue may connect with the inferior aspect of the skin. In addition, because nerve distributions in a meshed structure were also observed in the subcutaneous tissue (Fig. 6), this network of cutaneous nerve fibers may be considered to have direct influences along the long axis as well as along medial and lateral extensions ${ }^{4) 10}$.

The connective fibers between the subcutaneous tissue and fascia lata ran perpendicular to the horizontal plane. Based on this structure, it can be inferred that the connective fibers act as a stopper, thus preventing the movement of the positional transposition of the skin and its ligaments4)31). Skin ligaments or the retinacula cutis superficialis ${ }^{4) 31)}$ are usually almost perpendicular and present extensively in the face, hands, feet, and breasts ${ }^{37}$ ). In the present study, some fibers were found to be concentrated 


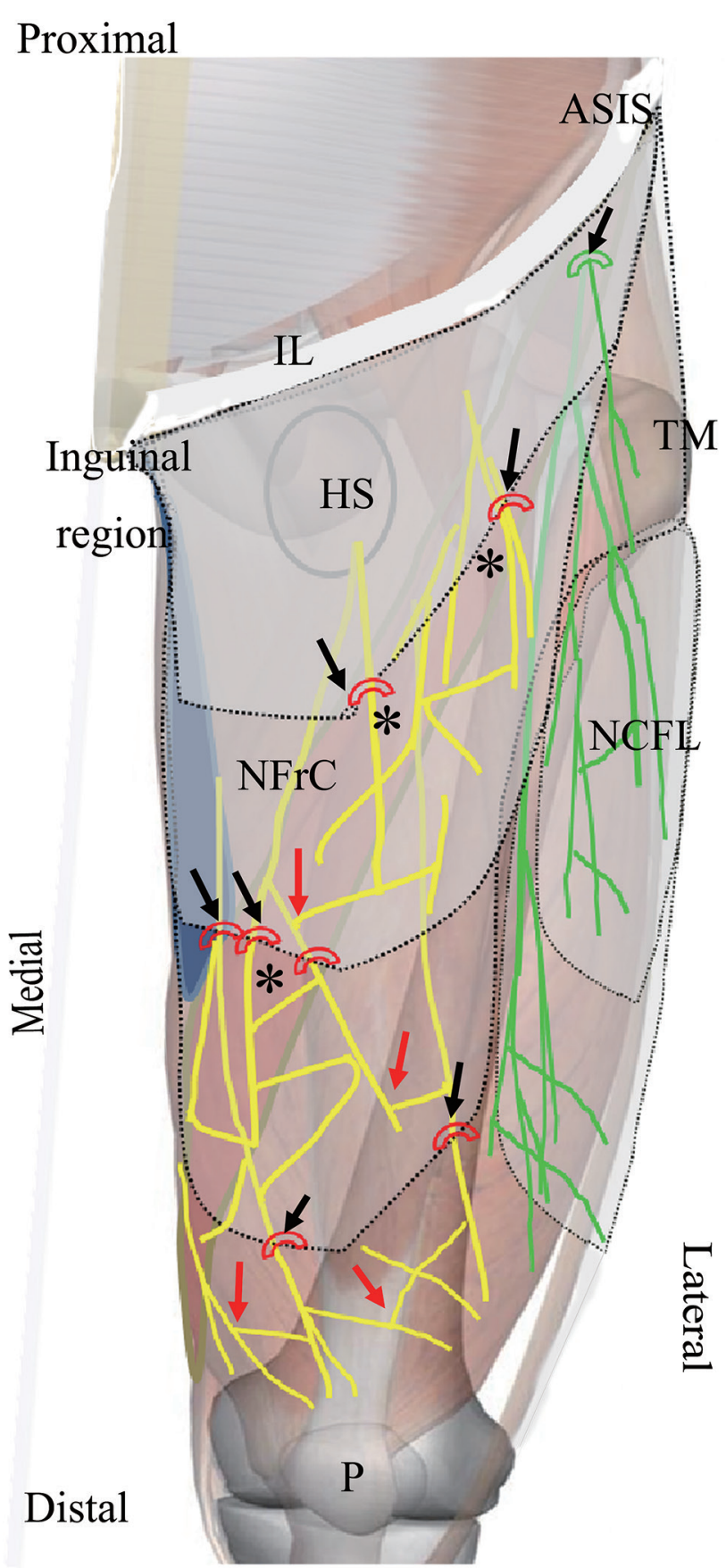

Fig. 6. Multilayered subcutaneous tissue and the cutaneous neural networks of the femoral nerve beneath the dermis. Cutaneous nerves (asterisks) emerge from the tunnel (black arrows) between the layers of subcutaneous tissue. Simultaneously with branching, they are anastomosed (red arrows) to other cutaneous nervous rami, forming a network of cutaneous nerves. Furthermore, there were sites where connective tissue fibers with nerves emerged in multiple directions toward the dermis. IL: inguinal ligament, HS: hiatus saphenous, TM: trochanter major, NCFL: nervus cutaneous femoris lateralis, $\mathrm{NFrC}$ : nervus femoris rami cutanei. in certain points from other directions and had various stratification patterns between the subcutaneous tissue and fascia lata. These fibers may be considered to be skin ligaments; if this is validated, it is possible that these skin ligaments, similar to fibers and cutaneous nerve networks, play a mutual role as the sensors of excessive tensile force applied to the skin.

According to Nagai ${ }^{38)}$, the stress of muscles is increased to induce hypertonia, resulting in the occurrence of pain sensations. An ultrasonic imaging device is useful for simple visualization and noninvasive determination of the structure of body surface. Cadaveric anatomical observation with ultrasonic imaging and open dissection revealed that the subcutaneous tissue comprised several layers at proximal levels in the thigh. These layers facilitate the visualization of the sites of fascial connections, which may reveal tightly bound connections between the subcutaneous tissue and fascia lata. If our findings in the cadavers are consistent with the anatomy in the living body, ultrasonic imaging could possibly be useful for the evaluation of a patient who has pain on motility.

\section{Conclusions}

The present study revealed the following findings: 1) the subcutaneous tissue of the thigh shows a structure comprising several layers; 2) the number of these layers decreases on progressing distally; 3 ) nerves in each layer were eventually distributed in the epidermis and dermis; and 4) the connective tissue in the thigh contains several skin ligament-like connective fiber bundles, which could constitute an element of motor restriction between the subcutaneous tissue and fascia lata while also encompassing cutaneous nerve fibers.

The important distinction of the present study involves a focus on the morphological structure of the subcutaneous tissue, which could provide data that are particularly beneficial in the field of sports/rehabilitation.

\section{Acknowledgements}

We express our hearty thanks to Ms. Yuki Ogawa and Mr. Koichi Koyama, staff persons of the Department of Anatomy, Tokyo Medical University, for their valuable support and encouragement for our studies. We would like to thank Prof. Yasushi Kobayashi of the Department of Anatomy, National Defense Medical College, for presenting us with an opportunity for preliminary observation of donated cadavers prior to the ultrasonic imaging observation. Also, we would like to thank Mr. Akihiro Umebara, a student of the Graduate School of Teikyo Heisei University, for his cooperation. 


\section{References}

1) Committee on Japan Anatomical Terminology; Termiologia Anatomica Japonica. Igaku-Shoin, Tokyo, 2007; 513pp

2) Kopsh FR; Integumentum Commune s. Cutis: in Rauber- Kopsh Lehrbuch und Atlas der Anatomie des Menschen. BandII. Georg Thieme Verlag Stuttgart, 1955; 680-731 (in German)

3) Bloom W, Fawcett, DW; Skin. In: A textbook of histology $10^{\text {th }}$ ed. W B Saunders Company, Phyladelphia, 1975; 563-597

4) Stecco C, Hammer WI; Subcutaneous tissue and superficial fascia. In: Stecco C, Hammer WI, eds, Functional atlas of the human fascial system. Churchill Livingstone Elzevier, London, 2014; $31-61$

5) Wendell-Smith CP: Fascia: an illustrative problem in international terminology. Surg Radiol Anat 1997; 19:273-277

6) Markman B, Barton FE Jr: Anatomy of the subcutaneous tissue of the trunk and lower extremity. Plast Reconstr Surg 1987; 80:284-54

7) Abu-Hijleh MF, Roshier AL, Al-Shboul Q, Dharap AS, Harris PF: The membranous layer of superficial fascia: evidence for its widespread distribution in the body. Surg Radio Anat 2006; 28:606-19

8) Warwick R Williams PL Eds.; The integument. In: Gray's Anatomy 35th ed., 1973; 1159-1169

9) Montagna W; The general anatomy of skin. In: the structure and function of skin, $2^{\text {nd }}$ ed. Academic Press, New York. 1962; 1-13

10) Ogawa $T$, Hosokawa $H$ : Integumentum commune. In:Kaibougaku Part 3, 10 th ed. Kanehara Shuppan, Tokyo, 1969; 67-92 (In Japanese)

11) Sinclair DC; Muscle and fasciae. In: Cunningham's textbook of anatomy. $11^{\text {th }}$ ed., Rolmanes G.J ed. Oxford University Press, London. 1972; 259-397

12) Kumka M, Bonar J: Fascia:a morphological description and classification system based on a literature review. J Can Chiorp Assoc 2012; 56:179-191

13) Myers $T$; Introduction: laying the railbed. In: Anatomy trains: myofascial meridians for manual and movement therapists, 3ed, Churchill Livingstone, Sydney, 2013; 1-11

14) Chaudhry H, Schleip R, Ji Z, Bukiet B, Maney M, Findley T: Three- dimensional mathematical model for deformation of human fasciae in manual therapy. J Am Osteopath Assoc. 2008; 108:379390.

15) Langer K: On the anatomy and physiology of the skin. Br J Plast Surg 1978; 31 (1): 3-8 (Translated from Langer, K.(1861). Zur Anatomie und Physiologie der Haut. I. Über die Spaltbarkeit der Cutis. Sitzungsbericht der Mathematisch-naturwissenschaftlichen Classe der Kaiserlichen Academie der Wissenschaften, 44, 19.).

16) Ottenio $M$, Tran $D$, Ní Annaidh $A$, Gilchrist $M D$, Bruyère $K$ : Strain rate and anisotropy efferacts on the tensile failure characteristics of human skin. J Mech Behav Biomed Mater 2015; 41:24150 .

17) Ní Annaidh A, Bruyère $K$, Destrade $M$, Gilchrist MD, Otténio $\mathrm{M}$ : Characterization of the anisotropic mechanical properties of excised human skin. J Mech Behav Biomed Mater. 2012; 5:139_ 48

18) Flynn C, Taberner AJ, Nielsen PM.; Characterizing skin using a three-axis parallel drive force-sensitive micro-robot. Conf Proc IEEE Eng Med Biol Soc. 2010; 2010:6481-4.

19) Ruvolo EC Jr, Stamatas GN, Kollias N: Skin viscoelasticity displays site- and age-dependent angular anisotropy. Skin Phar- macol Physiol. 2007; 20:313-21.

20) Monaco A, Grumbine NA: Lines of minimal movement. Clin Podiatr Med Surg. 1986; 3:241-7.

21) Kraissel CJ: The selection of appropriate lines for elective surgical incisions. Plast Reconstr Surg (1946). 1951; 8:1-28.

22) Borges AF: Relaxed skin tension lines. Dermatol Clin.1989; 7(1):169-77.

23) Fukui T: Skin taping (Hi-fu teiping). Publisher of Motion Medical "Undo to Igaku no Shuppannsha), Japan. 2014; 4-8 (in Japanese).

24) Li W, Ahn AC: Subcutaneous fascial bands-a qualitative and morphometric analysis. PLoS One. 2011; 6:e23987. doi:10.1371/ journal.pone.0023987.

25) Ahn AC, Kaptchuk TJ: Spatial anisotropy analyses of subcutaneous tissue layer: potential insights in its biomechanical characteristics. J Anat 2011; 219:515-24

26) Guimverteau J, Delage K, McGrouther D, Wong JK: The microvacuolar system: how connective tissue sliding works. J Hand Surg Eur 2010; 35:614-622.

27) Guimverteau J, Sentucq-Rigall J, Panconi B: Introduction to the knowledge of subcutaneous sliding system in humans. Ann Chir Plast Esth 2005; 50:19-34.

28) Kuruma H: Myofascial release. J Clin Sports Med 2013; 30:11631167 (in Japanese)

29) Ishii Y, Sakita M, Sasai N, Kasamatsu D, Komei S, Fukuda $\mathrm{T}$, Tsuchiya T: Functional role of fascia enveloping thigh and between muscles in the knee extensor of frog. Phys Ther Japan 2013; 40:16-23 (in Japanese with English abstract).

30) Lancerotto L, Stecco C, Macchi V, Porzionato A, Stecco A, De Caro R: Layers of the abdominal wall: anatomical investigation of subcutaneous tissue and superficial fascia. Sur Radiol Anat 2011; 33:835-42.

31) Nash LG, Phillips MN, Nicholson H, Barnett R, Zhang M: Skin Ligaments: regional distribution and variation in morphology. Clin Anat 2004; 17:287-293.

32) Nakajima $H$, Imanishi $N$, Minabe $T$, Kishi $K$, Aiso S:Anatomical study of subcutaneous adipofascial tissue: a concept of the protective adipofascial system (PAFS) and lubricant adipofascial system (LAFS). Scand J Plast Reconstr Surg Hand Surg. 2004; 38(5):261-6.

33) Kimura K, Takahashi, Y: Application of silver impregnation for peripheral nerve fiber analysis in topographic anatomy-a communicating branch of the lateral sural cutaneous nerve to the sural nerve in the crab-eating monkey. Acta Anat Nippon 1985; 60:8-10.

34) Takei H: Fascial manipulation. Manual physical therapy. 2014; 14:35-43 (in Japanese).

35) Kuruma H, Takei H, Tsurumi Y, Kurihara M: An approach for soft tissue thinking of muscle relations. J Clin Sports Med 2004; 30:13-16 (in Japanese).

36) Kuruma H, Takei H, Shida N, Kamio H, Yanagisawa K: Effects of myofascial release and stretching technique on range of motion and reaction time. J Phys Ther Sci 2013; 25:169-171.

37) Tsukahara K, Tamatsu Y, Sugawara Y, Shimada K: Relationship between the depth of facial wrinkles and the density of the retinacula cutis. Arch Dermatol 2012; 148:39-46.

38) Nagai S: Clinical condition kinematics of hip joint and physical therapy. (Kokansetsu no byoutai-undogaku to rigakuryoho I). J Phys Ther 2007; 24:362-374 (in Japanese). 